\title{
Urgences
}

\section{Corps défendant, à couvert, soi}

\section{Jean-Pierre Vidal}

Numéro 23, avril 1989

Lisière du livre

URI : https://id.erudit.org/iderudit/025511ar

DOI : https://doi.org/10.7202/025511ar

Aller au sommaire du numéro

Éditeur(s)

Urgences

ISSN

0226-9554 (imprimé)

1927-3924 (numérique)

Découvrir la revue

Citer cet article

Vidal, J.-P. (1989). Corps défendant, à couvert, soi. Urgences, (23), 12-21.

https://doi.org/10.7202/025511ar d'utilisation que vous pouvez consulter en ligne.

https://apropos.erudit.org/fr/usagers/politique-dutilisation/ 
De mots à mottes

Reliefs de l'arsenal de Roger Des Roches

Tout est normal, tout est terminé de Roger Des Roches

Monsieur Désir d'André Roy

La raie alitée d'effets d'André Gervais 


\section{JEAN-PIERRE VIDAL \\ Corps défendant, à couvert, soi}

Note sur les avatars phénoménologiques et historiques du péritexte de Reliefs de l'arsenal' de Roger Des Roches.

Pour André Gervais, poète musclé d'étoiles typographiques.

Espace de montre, espèce de trompe-l'oeil vestibulaire, la couverture toujours nous regarde et nous nomme, mieux encore, sirène, nous appelle: lecteurs, cochons de lecteurs. Voilà sans doute pourquoi la façon contemporaine exige de plus en plus quelque illustration en couverture, histoire de susciter la gamberge que le titre, élément devenu trop discret (nous, collectivement, ne savons plus guère lire ce qui n'a pas l'obscénité réconfortante de l'évidence), ne peut apparemment plus titiller. Et quand ce n'est pas une image adjuvante, c'est le titre lui-même, américainement magnifié jusqu'à dévorer la page couverture au complet, qui devient (hopefully) pur signal de salive monétaire.

II fut un temps, naguère, où les évidences ne nous étaient pas à ce point comminatoires. Un temps où l'ambiguîté séduisait les joueurs qu'étaient encore ces petits épargnants des régimes enregistrés de lecture capitalisation que nous sommes tous plus ou moins devenus. En ce temps-là, il y eut des folies douces, des présentations gentiment agressives: le Neige noire d'Aquin et ce livre de Des Roches, allez savoir pourquoi, la même année, faste donc sur ce plan au moins. Quatre ans plus tard, bien que noblement «composé(e) en Souvenir» (sic la Majuscule), l'édition Pierre Tisseyre du roman d'Aquin joue l'évidence rassurante: entre les deux mots qui composent le titre, formé de pointillés flocons noirs, une lisière d'effacement des lettres par un progressif surcroît de noir redonde visuellement l'oxymoron, atteignant ainsi une quasitautologie du verbal et de l'iconique qui, en bout de ligne, parce qu'elle «naturalise» la figure paradoxale du titre, en neutralise l'efficace. Douze ans plus tard (Des Roches n'a pas été canonisé. Stop. Purgatoire plus long. Stop.), l'édition qu'offrent «les herbes rouges" de Reliefs de l'arsenal témoigne d'un affadissement comparable. Sans doute le format revue et ses exigences de présentation, notamment une certaine standardisation, explique-til en partie cette déflation signifiante. Loin de moi, par ailleurs, l'idée d'accabler une maison d'édition qui est à bien des égards l'honneur des lettres québécoises. Peut-être enfin y a-t-il un certain droit, au 
moins moral, attaché à la maquette d'un livre et qui veut que l'on ne reprenne pas. II me semble, en tout cas, qu'il y a là surtout signe des temps, et d'autant plus que la dernière maquette est signée Des Roches. Les temps nous ont ici refait le coup de l' «obsolète", comme disent les Français, en anglais, comme d'habitude.

So let's go back to the future: la première couverture, muette, dévorante, énigmatique. Mais n'oublions pas, n'oublions jamais, sa descendance.

\section{Cependant un nez en moins fait guise en relief}

On connaît le dispositif créé par Jean Ricardou pour La prise/prose de Constantinople (1965). Une répercussion spéculaire qui fait de la quatrième de couverture un redoublement, une concurrence et un commentaire de la couverture. La maquette comme grammatologie. L'entrée triomphante de l'«Aufhebung» hégélienne dans la littérature française. En tout cas cela circule dès le paratexte que l'usage courant voudrait pourtant non seulement immobile mais même monumental.

Une même circulation, produit d'une comparable différence, produit même de la «différance» conceptualisée par Derrida, anime le premier Reliefs de l'arsenal, mur à mur, d'un couvert à l'autre.

Car, comme pour le roman de Ricardou, la couverture ici, d'entrée, se marque double. Mais ce n'est pas par le jeu de deux titres que différencie une lettre seule, le jeu sur la lettre sans lequel il n'y a pas vraiment de page de couverture (j'y reviendrai) se retrouvant ici, par ailleurs, à un autre niveau (y reviendrai itou), ce n'est pas par des inscriptions à proprement parler que se pose la duplication. C'est par l'ouverture d'un espace de légende et de nomination: celui qui dans le feuilleté du texte va, ô représentation!, ô mimésis!, d'une image à des mots. Que ce parcours ne doive être perçu, pensé, ni selon un axe téléologique, ni commme le simple tracé après coup d'une durée, mais comme une perspective, une profondeur de champ même, c'est ce que dit, me semble-t-il, l'optique au sens propre de ce dispositif dont le texte est dès lors l'image «virtuelle».

La couverture donc fait image. D'être réduite à une photographie tronquée de visage, sans inscription aucune, elle convoque la quatrième de couverture, non pas n'importe quelle, mais la qua- 
trième comme telle, espace habituel de la photo de l'auteur ${ }^{2}$. Une quatrième qui se pourrait dire négativisée dans la mesure où le portrait attendu, photo plus ou moins d'identité, est remplacé par une photo qui ne renvoie à rien sinon, bien sûr, et pour cela même, à elle-même. Dans la mesure aussi où aucun texte (biographique ou vendeur) ne l'accompagne. Dans la mesure encore où, tronquée, elle impose l'idée du collage, car la moindre coupure visible, en photographie, autonomise du coup chacun des éléments de l'image, tous devenus allogènes (si je puis, comme ici, enlever le nez d'un visage, c'est que je puis aussi avoir pris sans vergogne «les lèvres de n'importe qui ${ }^{3}$ et les yeux de quiconque, voire même ceux de l'enfance ${ }^{4}$ ). Dans la mesure enfin, mais c'est la première chose qui frappe, visible, dirait-on, "comme le nez au milieu de la figure», comme le nez absent ici de cette photo de couverture, dans la mesure où cette couverture est, dans tous les sens du terme, "déplacée».

Cette couverture est ainsi l'indice d'une absence par substitution, par métaphore (la couverture pour une autre, la photo pour une autre) et par soustraction (le texte et le nez «omis»), décomposition analytique, synecdoque. Par cela, elle vise et dissémine, elle est déictique et connotative, elle est déjà index de haute textualité. Elle devient donc, bien que n'en étant pas une, couverture par excellence, c'est-à-dire intertexte rendu visible. De tels détours pervers sont, pour qui connaît l'oeuvre, une des marques de commerce du holding RDR.

Mais dans l'univers des signes toute substitution est aussi, par le fait même, surenchère, ajout, surcroît. Si cette quatrième de couverture, négatif de couverture, couverture en trop, jaquette en quelque sorte, impose au lecteur qui s'en est saisi la recherche de la «vraie» couverture, plus loin, ailleurs déjà, de l'autre côté de l'objet livre, à la place instituée de la quatrième de couverture (il l'y trouvera) ou à l'intérieur, sur cette page de titre qui est traditionnellement la reprise de la page de couverture (ill'y trouvera aussi), en passant par le dos, lieu intermédiaire de saisie visuelle et manuelle du texte, espace ici où les inscriptions se lisent en une verticale ascendante ( ‘L'AURORE RELIEFS DE L'ARSENAL Roger DES ROCHES,$^{5}$ ) alors que ce qui est socialement inscrit là parcourt, comme il se doit, une verticale descendant $e^{6}$, auteur, titre, éditeur, si cette couverture déplacée, en trop, conduit à la manipulation physique du livre, exigée comme une cérémonie de dépouillement (dans tous les sens du terme), elle est aussi elle-même, dans son espace énigmatique, organisée selon une duplication qui en cache une autre, une de trop, comme tout simulacre, une de trop que 
justement l'absence (du nez) empêche de voir, évidente comme au milieu de la figure ainsi dessinée.

Car décidément ce visage sans nez, ce visage que, par cela même peut-être, on dirait déformé quand il est difforme, son axe est une pliure d'absence (équivalent iconique inverse - et même peut-être négativisé - de la tranche et de ses inscriptions qui forment l'axe du livre ouvert), son axe c'est l'effacement du nez, du relief. De tout relief, car il n'y manque pas que le nez. En effet, à y regarder d'un peu près on s'aperçoit que le tirage de la photo (ou, ce qui revient au même, sa reproduction sur la couverture) est tel qu'il manque aussi à ce visage saillies, ombres et méplats qui accusent toujours quelque relief facial aux portraits photographiques. Ce n'est ainsi plus un visage mais une collection d'organes et de traits plaqués. Bien entendu, dans cette convocation de surfaces, c'est la profondeur de l'identité qui se trouve menacée. Et d'autant plus que ce visage est ce masque où s'est de tout temps épandue la représentation de "l'éternelle ironie de la communauté " qu'Hegel assignait à la féminité. Visage ici, en effet, non pas de femme mais de féminité, le fardé se conjuguant au surexposé, aux reliefs gommés, pour dénier dans le simulacre toute vérité biologique de sexe. Platitude exemplairement décevante de la couverture réduite à un visage sans identité, même générique, une visage dont les traits ne sont que des marques reportées, déportées.

Et c'est précisément parce qu'il n'y a ici rien d'autre que le cadre et son arbitraire qui puisse tenir lieu de désignation unificatrice, c'est parce que ce visage est une condensation arcimboldesque qu'une rime contagieuse s'installe dans cet espace piégé, une rime qui déstabilise l'image, la fait imploser au point que la lecture tout entière à cette duplication d'yeux, de lèvres, oublie, dans son incessant effort pour unifier malgré tout ce puzzle en semblant de face humaine, de remarquer qu'il y a là deux sourcils de trop.

Deux charbonneux, deux macules de fard comme des lèvres désunies qui viennent coiffer les sans doute rasés, à peine fardés que l'on voit juste au dessus des yeux.

Et maintenant la couverture n'est plus, par ce surcroît, par ce fard de fard, qu'un collage de parenthèses ouvertes et d'accolades décollées, de demi-cercles et de paraboles, entourant deux yeux tristes qui nous fixent, comme une supplication de freak qui ferait la retape. 
Sil'on passe outre enfin cette couverture, le rabat intérieur de cette même couverture nous donne, comme prime de feuilletage et mime de lecture, la vérité provisoire du déplacement: le syntaxique et le métonymique révélés comme un au-delà transitoire du métaphorique et du paradigmatique, qui, je crois l'avoir montré, ordonnaient la page de couverture. Indice de syntaxe en effet que ce nez qui nous saute ici aux yeux, ce nez surmonté d'un seul oeil, celui, semble-t-il, qui était à droite de l'image de couverture, mais se trouve ici inversé, la paupière manifestement inférieure mise ici en position supérieure.

Néanmoins donc, ce Cyclope, à vue de nez, louche (et j'écris cela dans tous les sens que me permet cette langue devenue folle d'être restée «in cheek», enfermée dans un couvert muet).

Interlope décidément le monde de ce texte où nous voici comme malgré nous entré, passées les lèvres de couverture, à la rencontre du "péritexte éditorial» (Genette), dans l'ordre: 1) “Reliefs de l'Arsenal est le premier volume de la "collection écrire" des Éditions L'Aurore». 2) “Collection dirigée par André Roy». 3) «RELIEFS DE L'ARSENAL», le tout réparti sur trois (3) pages et dans la typographie que j'ai ici respectée quant au gras et aux capitales, le tout avant la page de titre avec laquelle il fait système. Le tout, fameuse médecine. So, now...

\section{How do you spell relief?}

C'est bien, en effet, la question qui se pose. Et, de même que pour la couverture et son rabat, elle se pose dans l'horizontalité d'une ligne où la succession des espaces de pages ne fait que souligner une syntaxe qui est maintenant celle du récit. Le récit tout d'abord de l'épiphanie du texte comme spécificité, fût-elle titrée, signée.

En effet, du premier "Reliefs de l'Arsenal», émergeant en gras d'un énoncé qui l'inclut dans une série éditoriale dont il est la première manifestation, titre "rhématique" (Genette) alors, au second en capitales, pas tout à fait encore "thématique», car il lui manque les marques génériques qui seules pourront le faire fonctionner comme l'index de la fiction, marques qui se retrouveront un peu plus loin, comme il se doit, dans la page de titre, du premier au second «relief» donc, c'est toute une cartographie paradigmatique qui se met en place. S'y déploie l'espace nébuleux du signifiant. Relief est d'abord "ce qui fait saillie sur une surface" (Robert) 
avant d'être surtout «ce qu'on enlève d'une table servie» (Robert encore), un reste.

Un tel signifiant représente bien sûr, dans la polarité qui s'institue entre ses deux groupements de sens, une des plus exactes métaphores de l' "écriture", dans l'acception derridienne qui la dit essentiellement supplément et trace. Mais, ce qui est encore plus éclatant, l'écriture se dit ici «relief» de façon spatiale, iconique, typographique (doublement: le premier «relief» pose en effet le «type» dont il est le, en toutes lettres, le «token») et topographique, sans cesser un seul instant d'être tropographique, par définition.

Le premier «relief», en saillie typographique, s'illustre luimême tout en servant de légende au manque dans le visage de la page de couverture. Le deuxième est l'amplification de ce processus, redoublement et capitalisation, en même temps que sa négation: comme le nez du rabat, il est reste et supplément de la formule qui l'incluait dans une collection à venir, reste et supplément vite devenus pure superfluité dès que se tourne la page sur laquelle il se marque, car voici, apparaissant enfin dans l'effacement rétroactif de tout ce qui précède, la vérité monumentale et funéraire de la page de titre.

La nouvelle couverture des «Herbes rouges» qui donne d'entrée de jeu, sur la même surface, tout ce déploiement ${ }^{7}$, et qui joue d'abord et avant tout la référence, la collection, la série, jusqu'à inclure une méchante illustration de S.F. américaine des années cinquante, rétro, post-moderne, voire carrément kitsch, cette nouvelle couverture scelle ainsi le sort du texte: l'objet sans nom qui émergeait au jour de soixante-quatorze a fini par rentrer dans la bibliothèque, la collection dont il était l'indice prophétique s'est refermée sur lui. À côté, sur les rayons, il y a toutes les oeuvres de RDR... et celles d'A.R., André Roy.

Car la littérature (et quelle maison québécoise pourrait voir plus de titres à représenter celle de ces années-là que «les herbes rouges"?) donne un nom et des lettres de noblesse à un prénom, un prénom que l'oeuvre secrètement épelle.

\section{Roger}

C'est ce que dit, cinq sur cinq, le pilote; ici d'ailleurs, dans cette version française de ce vieux film de guerre des années 
cinquante, le pilote, un pote, est un prote qui assemble des tropes. Car avant d'être le brillant éditeur et concepteur de logiciels que l'on connaît, RDR fit des études de graphisme (cf. la maquette de son biplan quadrimoteur, dont je viens ci-haut de parler) et devint typographe.

Nous voici donc en pleine biographie, en plein nom.

Et d'abord dans l'absence. Car c'est ce qui frappe aussi dans cette première édition. L'absence visible de tout nom sur la couverture. Cette absence n'est pas réductible, ni même comparable, à l'absence de toute autre marque scripturale. Car si l'image peut, à la limite, tenir lieu de titre, précisément parce qu'elle est d'emblée un rébus, si elle peut, parce qu'elle est regard qui fixe le lecteur et l'appelle, remplacer l'éditeur et tout ce qui s'y rapporte dans la rhétorique sociale de l'échange qu'exemplairement elle met en scène, rien en revanche sur cette couverture n'indique la filiation, l'identité, nulle signature, griffe sur la mondanité de cet objet, ce calme bloc ici-bas chu d'un désastre obscur (lui murmure Stéphane). Au contraire, comme on l'a vu, c'est précisément l'identifé qui se trouve déniée, et à plus d'un titre, comme je crois l'avoir montré, sur cette surface de condensation.

Non, le premier nom du livre sera celui du directeur de la collection: André Roy. Et il est en bout de ligne, vraiment en bout de ligne puisque d'abord c'est la collection qui est dite sur la première page et que ce n'est qu'au verso de cette même page que ladite collection est affirmée «dirigée par André Roy». Comme si le gras voulait ici dire amovible. Dans la permanence de la collection qui pourtant ici se fonde, se soutient de son futur, d'un futur qu'elle ne fait pourtant que prédire (belle «déclaration d'indépendance» à la Derrida $^{8}$ ), une collection qui a par ailleurs le front de s'appeler tout simplement «écrire», injonction, futur encore, dans cette permanence qui se dilate vers une sorte d'éternité, le nom est un relief qu'un rien peut emporter.

C'est que le nom est toujours un peu formé de minuscules labiles, passé l'étai capital des majuscules à sa double initiale. Et si «Reliefs de l'Arsenal», en sa première occurrence, rime avec «Éditions (de) l'Aurore», à l'aube de cette collection qu'il inaugure, comme si "éditions» ne pouvait s'écrire que "reliefs", André Roy en reprend, inversées, les majuscules... puisque, justement, il dirige la collection, est-on tenté d'écrire. 
On ne saurait, on le voit, jouer trop longtemps le syntaxique qu'un effet paradigme, le volume, le coffre du texte, ne s'en reforme. La quatrième de couverture a, elle aussi, un rabat et dessus, en guise de nez peut-être ${ }^{9}$, un texte, «prière d'insérer» et manifeste en même temps puisque les caractéristiques et qualités qu'il attribue à RDA sont sans doute celles qu'il voudra(it) voir aux plats mitonnés ou aux excroissances dressées par le chef qui signe "a.r.", en minuscules s'il vous plaît, et après un autre signataire: «r.d.r.» Ce que signent les deux compères, c'est, entre autres, ceci: «Et M-, personnage principal? Il échappe comme tous les autres à un étiquetage définitif, à une identification par ses dimensions «humaines». Dans le texte, $\mathrm{M}$ - - chaque personnage et le narrateur ne sont pas nommés, plutôt calqués (l'un sur l'autre) jusqu'à être interchangeables, flexibles..."

Venons-en donc enfin à la page de titre qui est la réplique exacte de la quatrième de couverture, "écrire" et sa barre exceptés (parbleu! Ils sont, nous venons de le voir, sur le rabat puisque le texte r.d.r. / a.r. ne parle sans doute que de ça), une page de titre où, par l'accident réglé des lettres, les noms ne sont que des mots "calqués (l'un sur l'autre) jusqu'à être interchangeables, flexibles».

Et il est temps maintenant de dire que la lettre est toujours ce qui se met en scène sur toute couverture. À cause de sa raréfaction visible et aussi, historiquement, parce que c'est la séparation même du nom de l'auteur et du titre d'avec le reste de l'incipit, c'est ce décollage social qui a produit la couverture telle que nous la connaissons, la couverture comme verticalité de montre surgie en lieu et place du déroulement du parchemin où les noms propres du texte se perdaient dans l'anonymat du littéral. Cette verticalité, j'ai déjà dit que la couverture de ce texte en joue iconiquement, comme d'ailleurs en jouait littéralement la couverture de Neige noire $^{10}$.

Or, si la lecture est sans doute plus souvent horizontale ou oblique, elle se donne aussi pour verticale, probablement à cause de la superposition des lignes. Elle «descend» toujours et, en page de couverture ou de titre, l'auteur, dans ses hauteurs, est un dieu qui profère un titre, comme négligemment l'on baptise quand on est Adam. L'horizon terrestre commence au générique, «roman», "récit", "poème», etc., mode d'emploi, évangile à totons. Plus bas encore, l'éditeur assume le rôle ambigu d'un Hadès qui s'est laissé séduire et grâce à qui, de son obscurité surgi, l'auteur, de ses hauteurs dès lors, est un dieu en état perpétuel de renaissance qui 
jette négligemment un titre, comme Adam et tout ce qui s'ensuit à I'horizon lecteur.

Or ici, et un peu selon la même stratégie que celle de la couverture exclusivement iconique avec laquelle cette page de titre forme système, le dieu est descendu à l'horizon de la lecture grâce au leurre des rimes, cette fois-ci littérales. Encore faut-il aussitôt préciser que ce littéral-là prend son sel d'une manière d'iconicité: la typographie et son jeu. Car «roger des roches» et "reliefs de l'arsenal» sont ici inscrits dans les même caractères (simplement plus gros et en gras pour le titre), sans majuscule d'aucune sorte ni de quelque allégeance.

Aquin jouait la verticale plane, Des Roches mise ici sur la partition horizontale croisant la chute obligée de la lecture à la verticale sociale. C'est le jeu déjà relevé des trois ou quatre feuilletés de la «page de couverture». C'est aussi le spéculaire des inscriptions: roger des roches, reliefs de l'arsenal, le tout en bas de casse, et, en quatrième de couverture, écrire/l'aurore (souligner la barre de (ré)partition, voilà bien une nécessité du discours critique qui pourrait servir d'emblème au texte). Et l'on pense aussitôt à quelque lapsus inévitable: rocher des roches, fameux relief tout ça de quelque arsenal résolument métonymique, puisque de toute façon dans cet incipit redevenu manuscrit le «reliefs" du titre n'est que l'apposition, le filé des «roches» du nom. «Roger» dit le pilote qui a trop vu de films américains.

Car même si la cérémonie de l'inscription qui, dans le feuilleté des trois pages où il apparaît répercuté mais divers, de premier exemplaire d'une collection à ultime avatar d'une signature, semble bien faire du titre une permanence identitaire, son tracé provisoirement final au fronton de la page de titre où ses lettres se mêlent à celles de qui s'y est perdu, disséminé, auteur, dit au contraire, dans le monumental de la verticale que tout texte est cénotaphe et que rien, jamais, n'aura eu lieu qui soit son nom propre ou son éponyme en cette constellation sociale que G.G. nomme «paratexte».

1. D'abord paru aux Éditions L'Aurore, coll. "Écrire», en 1974, ce texte a été récemment repris par "Les herbes rouges", no 145-146, en 1986. Le lecteur attentif remarquera, outre une différence sans doute inévitable dans la page de couverture, différence sur laquelle je reviendrai, un certain nombre de corrections, souvent même de précisions, dans la nouvelle édition, corrections et précisions qui ont toutes pour effet de rendre le texte plus lisible, moins opaque. Nous ne sommes plus dans les années soixante-dix. Mais que dire alors des résistances, des impropriétés, voire des agrammatismes qui ont été 
soigneusement gardés? L'espace dont je dispose ici ne me permettant pas de trop m'étendre là-dessus, je me bornerai à dire que j'y vois d'une part une obscurité de surcroît par rapport à la première version, d'autre part une très mallarméenne ou duchampienne capture du hasard faiseur de ready-mades scripturaux. Élevage de poussière de lettres, R. Mutt, c'est-à-dire, selon la lecture phonétique et translinguistique: "art bâtard".

2. Le paratexte de Neige noire tire aussi divers effets de la subversion de cette quatrième de couverture canonique. $\mathrm{Cf}$. à ce sujet mon article dans Études et travaux, Grenoble, no 20, 1981, en particulier p. 56-57.

3. Titre d'un texte en prose de RDR («Les herbes rouges", no 70, 1978).

4. L'enfance d'yeux, titre d'un recueil de poèmes du même RDR (Éditions du Jour, 1972) où l'on voit précisément à l'oeuvre l'inversion d'un cliché langagier: celui que j'ai reformé. L'optique du soma, tel pourrait être le sous-titre de toute l'oeuvre à ce jour publiée. Une oeuvre qui travaille, peut-être plus que toute autre, ce lieu de la «jonction» du symbolique et de l'imaginaire dont parle Lacan (cf. Le séminaire, Livre 1, Seuil, Paris, 1975. Notamment «la topique de l'imaginaire», p. 85-182).

5. La distribution capitale/bas de casse, que j'ai ici scrupuleusement respectée, a son importance. J'y reviendrai.

6. La boucle ainsi formée empêche cette tranche d'être vraiment l'équivalent de la page de couverture dans la mesure où le nom de l'auteur pourtant présent à sa place attitrée, en haut, ne peut se lire qu'en dernier, en partant du bas. L'impression selon une verticale descendante n'aurait évidemnent pas permis cet effet paradoxal de «double bind".

7. Ce faisant, bien sûr, elle nie ce déploiement et c'est en quoi, seconde, elle en est le commentaire, mieux, l'épitaphe.

8. Cf. Otobiographies. L'enseignement de Nietzsche et la politique du nom propre, Paris, Galilée, 1984.

9. C'est-à-dire en guise de manque du texte de son verso: la page de couverture déportée en quatrième. Mode d'emploi ou glose de cette étrange et laconique appellation: "RÉCIT» qui surplombe cette non moins étrange combinaison "ÉCRIRE / L'AURORE". La barre n'y pourra rien, il se lit une fameuse formule de Giraudoux là-dedans: "Cela a un très beau nom, femme Narsès, cela s'appelle l'aurore" (phrase finale d'Électre).

10. Cf. article cité, note 2 . 